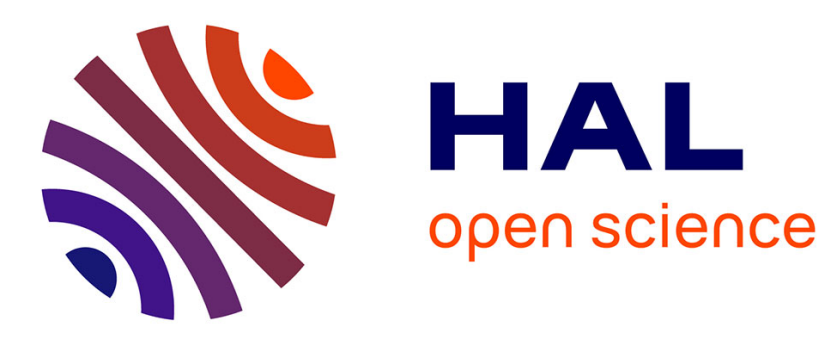

\title{
Upwind Finite Volume Schemes for One and Two Dimensional Euler Equations
}

Thierry Gallouët, Jean-Marc Hérard, Raphaèle Herbin, Jullian Nicolas, Nicolas Seguin

\section{- To cite this version:}

Thierry Gallouët, Jean-Marc Hérard, Raphaèle Herbin, Jullian Nicolas, Nicolas Seguin. Upwind Finite Volume Schemes for One and Two Dimensional Euler Equations. 16th AIAA Computational Fluid Dynamics Conference, Jun 2003, Orlando, United States. 10.2514/6.2003-4122 . hal-01583877

\section{HAL Id: hal-01583877 \\ https://hal.science/hal-01583877}

Submitted on 4 Dec 2019

HAL is a multi-disciplinary open access archive for the deposit and dissemination of scientific research documents, whether they are published or not. The documents may come from teaching and research institutions in France or abroad, or from public or private research centers.
L'archive ouverte pluridisciplinaire HAL, est destinée au dépôt et à la diffusion de documents scientifiques de niveau recherche, publiés ou non, émanant des établissements d'enseignement et de recherche français ou étrangers, des laboratoires publics ou privés. 


\title{
UPWIND FINITE VOLUME SCHEMES FOR ONE AND TWO DIMENSIONAL EULER EQUATIONS
}

\author{
Thierry Gallouët ${ }^{1, *}$, Jean-Marc Hérard ${ }^{1,2, \dagger}$ Raphaele Herbin ${ }^{1, \ddagger}$ \\ Nicolas Jullian ${ }^{1, \S}$, Nicolas Seguin ${ }^{1, \uparrow}$ \\ ${ }^{1}$ Université de Provence, Centre de Mathématiques et d'Informatique, \\ Laboratoire d'Analyse, Topologie et Probabilités - UMR 6632, \\ 39 rue Joliot Curie, 13453 Marseille cedex 13, FRANCE \\ gallouet@cmi.univ-mrs.fr, herard@cmi.univ-mrs.fr, herbin@cmi.univ-mrs.fr \\ jullian@cmi.univ-mrs.fr, seguin@cmi.univ-mrs.fr \\ 2 Électricité de France, Division Recherche et Développement, \\ Département Mécanique des Fluides et Transferts Thermiques, \\ 6 quai Watier, 78401 Chatou cedex, FRANCE \\ herard@chi80bk.der.edf.fr
}

\begin{abstract}
The true rate of convergence of some Finite Volume upwinding techniques is examined herein, when focusing on Euler equations with an arbitrary equation of state (EOS), in one and two space dimensions. Standard conservative explicit schemes based on exact and approximate Godunov schemes tend to yield unacceptable results on coarse meshes, although they converge when the meshes are refined. Here, a new hybrid scheme is presented, which provides rather accurate results on coarse meshes, unlike previous schemes. We also show that perfect representation of unsteady contact discontinuities requires adapting the mesh interfaces in such a way that slip lines are tangent to the cell interfaces, but also that a slightly modified version of the conservative scheme may be used when strong shear flows are computed on coarse meshes.
\end{abstract}

\footnotetext{
${ }^{*}$ Professor

†Senior Engineer in EDF, Associate Research Director at CNRS -UMR CNRS 6632-, LATP, Université de Provence, AIAA member, corresponding author

$\ddagger$ Professor

$\S \mathrm{PhD}$ student

I Associate Professor

Copyright (C) 2003 The American Institute of Aeronautics and astronautics, Inc. All rights reserved.
}

\section{INTRODUCTION}

Contact discontinuities occur in various CFD convective systems. This is true of course for Euler gas dynamics, but also when investigating two phase flows with the help of homogeneous models ( $\left.\mathrm{see}^{2},{ }^{4},{ }^{7},{ }^{6},{ }^{9}\right)$, or with so-called two-fluid models. Quite recently, some works related to the two-fluid approach also suggest not to assume instantaneous pressure relaxation; this may result in the appearance of several contact discontinuities $\left(\operatorname{see}^{5},{ }^{18},{ }^{10}\right)$. This is also true when investigating statistical turbulence with the help of Reynolds stress closures in single phase flow. The problem is that the contact discontinuities induce a weak rate of convergence even for $1 D$ flows, when using standard conservative schemes. This will be briefly recalled in the first part of the paper, though the reader may refer to ${ }^{13}$ for more details on the basic procedure. Troubles become even worse in $2 D$ simulations where the number of cells is reduced due to CPU constraints, but also due to the obvious fact that the mesh interfaces may not be aligned with all the plane discontinuities -as automatically occurs in the $1 D$ framework-. As a consequence, when focusing on the Euler equations for instance, it immediately appears that remedies obtained in a one dimensional framework are not necessarily sufficient in the $2 D$ 
framework due to the occurence of shear effects.

The aim of the present paper is thus to provide some tools to deal with Euler equations with any equation of state (EOS) on any type of mesh, assuming an arbitrary highly sheared medium. Schemes discussed herein rely on the exact Godunov scheme ${ }^{16}$ and also on some particular approximate Godunov schemes derived from the VFRoe-ncv formalism with use of $Y^{t}=(\mathbf{U}, P, g(\rho, s), C, \psi)$ variables ${ }^{8},{ }^{12}$, see notations below. We start by recalling the basic ideas initially proposed $\mathrm{in}^{13}$ when restricting to the $1 D$ framework. These contain previous proposals by Abgrall ( ${ }^{1}$ ) when restricting to stiffened gas EOS. We then turn to the $2 D$ framework, and propose three distinct numerical procedures to compute approximate solutions. The first one relies on the basic conservative scheme. The second one is a -straightforward- extension based on the work described in $^{13}$ in the $1 \mathrm{D}$ framework. The third one is new and aims at improving accuracy on coarse unstructured $2 \mathrm{D}$ meshes.

\section{D GOVERNING EQUATIONS AND EOS}

We first start recalling some results obtained in a purely one dimensional framework. Using standard notations, $W=(\rho, \rho C, \rho U, E, \rho \psi)$ stands for the conservative variable and $F(W)=$ $\left(\rho U, \rho C U, \rho U^{2}+P, U(E+P), \rho \psi U\right)$ denotes the physical flux in the $1 \mathrm{D}$ framework. The set of equations reads:

$$
\left\{\begin{array}{l}
\frac{\partial W}{\partial t}+\frac{\partial F(W)}{\partial x}=0 \\
W(x, 0)=W_{0}(x)
\end{array}\right.
$$

( $\rho$ and $P$ stand for density and pressure respectively, $U$ denotes the mean velocity, $Q=\rho U$ is the momentum, $C$ is the mean concentration of a pollutant, $E$ is the total energy and $\psi$ is the colour function). The speed of acoustic waves is noted $c$. We also need to provide suitable boundary conditions. A physically relevant entropy inequality selects admissible shocks, and requires introducing the specific entropy $s(P, \rho, C, \psi)$. The total energy is written in terms of the kinetic energy plus the internal energy $\rho e: E=\rho U^{2} / 2+\rho e$, where $\rho e=\phi(P, \rho, C, \psi)$. We then introduce classes $T_{1}$ and $T_{2}$ which contain EOS which respectively take the form:

$$
\begin{aligned}
& \rho e=\phi_{1}(P, \rho, C, \psi) \\
& =\rho\left(a_{1}(P)+b_{1}(P) C+c_{1}(P) \psi\right)+d_{1}(P) \\
& \rho e=\phi_{2}(P, C, \psi) \\
& =f_{2}(C, \psi) h_{2}(P)+g_{2}(C, \psi)
\end{aligned}
$$

We recall here that both $C, \psi$ are both transported with velocity $U$, and thus are solutions of the first equation of (13). For any EOS, the internal energy may be decomposed in terms of contributions in $T_{1} \cup T_{2}$ and a remaining part if necessary:

$$
\begin{aligned}
& \rho e-\phi_{1}(P, \rho, C, \psi)-\phi_{2}(P, C, \psi) \\
& =\phi_{3}(P, \rho, C, \psi)
\end{aligned}
$$

The perfect gas single component EOS, the Tait EOS and the Tamman law belong to $T_{1}$. The mixture of perfect gases or equivalently the stiffened gas EOS lie in $T_{2}$ exactly. Obviously, the MieGrüneisen EOS belongs to $T_{3}$. We emphasize that if the EOS lies in $T_{1}$, the standard conservative Godunov scheme (or the conservative approximate Godunov scheme VFRoe-ncv with the above mentionned variable $Y$ ) will perfectly represent unsteady contact discontinuities on any mesh. Indeed, when using the standard algorithm, the cell value of pressure (which is used to initialize the local Riemann problem at the interface between cells $i$ and $(i+1))$ is given by $p_{i}^{n}=P_{i}^{n}$ solution of:

$$
\phi\left(P_{i}^{n}, \rho_{i}^{n}, C_{i}^{n}, \psi_{i}^{n}\right)=E_{i}^{n}-\frac{\left(Q_{i}^{n}\right)^{2}}{2 \rho_{i}^{n}}
$$

Introducing standard notations for the time step $\delta t$, the mesh size $h_{i}=x_{i+1 / 2}-x_{i-1 / 2}$, let us denote $W\left(Y_{x_{i+1 / 2}}^{*}\right)$ the interface value predicted by the exact or approximate Godunov scheme at $x_{i+1 / 2}$, then the mean value of the conservative variable $W$ over cell $i$ at time $k \delta t$, namely $W_{i}^{k}$, which is obtained through:

$$
\begin{aligned}
& h_{i}\left(W_{i}^{n+1}-W_{i}^{n}\right)= \\
& -\delta t\left(F\left(W\left(Y_{x_{i+1 / 2}}^{*}\right)\right)-F\left(W\left(Y_{x_{i-1 / 2}}^{*}\right)\right)\right)
\end{aligned}
$$

satisfies the property of "perfectly preserving the unsteady contact discontinuity" as defined below. Note that in the case of an exact Godunov scheme, we have formally $Y(W)=W$.

Definition 1: Contact discontinuities are perfectly preserved by a first-order three-point scheme, if, for any values of density, concentration and colour, and for any velocity and pressure satisfying: $U_{k}^{n}=U_{0}$ and $p_{k}^{n}=P_{0}$, for $k=i-1, i, i+1$, then the computed velocity and pressure at time $t^{n+1}$ satisfy: $p_{i}^{n+1}=P_{0}$, and $U_{i}^{n+1}=U_{0}$. 


\section{HybRid Godunov SCHEME IN A 1D \\ FRAMEWORK}

We first present the hybrid scheme, which is valid for any EOS, but is no longer fully conservative. In this hybrid scheme, we no longer use $p_{i}^{n}=P_{i}^{n}$, where $P_{i}^{n}$ issues from (5). For any EOS which does not lie in $T_{1}$, we set: $g_{0}(C, \psi)=$ $f_{2}(C, \psi) h_{2}\left(P_{r e f}\right)+g_{2}(C, \psi)$ (the only useful values will be $h_{2}\left(P_{\text {ref }}\right)=0$ and $h_{2}\left(P_{\text {ref }}\right)=1$ ) The first-order scheme is, setting $\gamma=\rho c^{2} / P$ :

$$
\begin{aligned}
& h_{i}\left(W_{i}^{n+1}-W_{i}^{n}\right) \\
& \quad+\delta t\left(F\left(W\left(Y_{x_{i+1 / 2}}^{*}\right)\right)-F\left(W\left(Y_{x_{i-1 / 2}}^{*}\right)\right)\right)=0 \\
& h_{i}\left(\left(g_{0}\right)_{i}^{n+1}-\left(g_{0}\right)_{i}^{n}\right) \\
& \quad+\delta t \hat{U}_{i}\left(g_{0}\left(Y_{x_{i+1 / 2}}^{*}\right)-g_{0}\left(Y_{x_{i-1 / 2}}^{*}\right)\right)=0 \\
& h_{i}\left(\left(\phi_{3}\right)_{i}^{n+1}-\left(\phi_{3}\right)_{i}^{n}\right) \\
& \quad+\delta t \hat{U}_{i}\left(\phi_{3}\left(Y_{x_{i+1 / 2}}^{*}\right)-\phi_{3}\left(Y_{x_{i-1 / 2}}^{*}\right)\right) \\
& \quad+\delta t \hat{H}_{i}\left(U_{x_{i+1 / 2}}^{*}-U_{x_{i-1 / 2}}^{*}\right)=0 \\
& 2 \hat{U}_{i}=U_{x_{i+1 / 2}}^{*}+U_{x_{i-1 / 2}}^{*} \\
& 2 \hat{H}_{i}=\left(\gamma P \frac{\partial \phi_{3}}{\partial P}+\rho \frac{\partial \phi_{3}}{\partial \rho}\right)\left(Y_{x_{i-1 / 2}}^{*}\right) \\
& \quad+\left(\gamma P \frac{\partial \phi_{3}}{\partial P}+\rho \frac{\partial \phi_{3}}{\partial \rho}\right)\left(Y_{x_{i+1 / 2}}^{*}\right)
\end{aligned}
$$

The definition of the numerical flux is:

$$
\begin{aligned}
& F\left(W\left(Y^{*}\right)\right)=\left(\rho^{*} U^{*}, \rho^{*} U^{*} C^{*}, \rho^{*}\left(U^{*}\right)^{2}+P^{*}\right. \\
& \left.U^{*}\left(\rho^{*}\left(U^{*}\right)^{2} / 2+P^{*}+(\rho e)^{*}\right), \rho^{*} U^{*} \psi^{*}\right) \\
& (\rho e)^{*}=\phi_{1}\left(P^{*}, \rho^{*}, C^{*}, \psi^{*}\right)+f_{2}\left(C^{*}, \psi^{*}\right) h_{2}\left(P^{*}\right) \\
& +g_{2}\left(C^{*}, \psi^{*}\right)+\phi_{3}\left(P^{*}, \rho^{*}, C^{*}, \psi^{*}\right)
\end{aligned}
$$

Both sequences $\left(f_{2}\right)_{i}^{k}$ and $\left(g_{2}\right)_{i}^{k}$ are computed setting $g_{0}$ equal to $h_{2}\left(P_{\text {ref }}\right)=0$ and to $h_{2}\left(P_{\text {ref }}\right)=1$ successively. The cell pressure used to compute the local Riemann problems at the beginning of the next time step, namely $p_{i}^{n+1}=\tilde{P}_{i}^{n+1}$, is obtained by computing the solution $\tilde{P}_{i}^{n+1}$ of equation:

$$
\begin{aligned}
E_{i}^{n+1}-\frac{\left(Q_{i}^{n+1}\right)^{2}}{2 \rho_{i}^{n+1}}= & \phi_{1}\left(\tilde{P}_{i}^{n+1}, \rho_{i}^{n+1}, C_{i}^{n+1}, \psi_{i}^{n+1}\right) \\
& +\left(f_{2}\right)_{i}^{n+1} h_{2}\left(\tilde{P}_{i}^{n+1}\right)+\left(g_{2}\right)_{i}^{n+1} \\
& +\left(\phi_{3}\right)_{i}^{n+1}
\end{aligned}
$$

with given values $E_{i}^{n+1}, Q_{i}^{n+1}, \rho_{i}^{n+1}, C_{i}^{n+1}, \psi_{i}^{n+1}$ provided by the discrete conservative equations (7), and $\left(f_{2}\right)_{i}^{n+1},\left(g_{2}\right)_{i}^{n+1},\left(\phi_{3}\right)_{i}^{n+1}$ provided by discrete non-conservative equations $((8),(9))$. The resulting value of the cell pressure is used to initialize the Riemann problems at each cell interface at the beginning of the following time step. Discrete equations (8-9) provide numerical approximations of solutions of the non conservative governing equations:

$$
\begin{aligned}
& \frac{\partial g_{0}}{\partial t}+U \frac{\partial g_{0}}{\partial x}=0 \\
& \frac{\partial \phi_{3}}{\partial t}+U \frac{\partial \phi_{3}}{\partial x}+\left(\gamma P \frac{\partial \phi_{3}}{\partial P}+\rho \frac{\partial \phi_{3}}{\partial \rho}\right) \frac{\partial U}{\partial x}=0
\end{aligned}
$$

The latter two provide redundent information for regular solutions. Note that, for Chemkin EOS or for some complex analytic laws, the CPU time required to compute $\tilde{P}_{i}^{n}$ is lower than the CPU time for $P_{i}^{n}$. It is also important to note that when both $\phi_{2}$ and $\phi_{3}$ are null, the cell pressure $\tilde{P}_{i}^{n}$ computed using (12) identifies with the standard cell value of pressure $P_{i}^{n}$ issuing from (5). Obviously in that case, one does not need to compute equations (8-9). Unsteady contact discontinuities are now perfectly represented for any mesh size since we have:

Property 1: For any EOS, the modified Godunov or the present VFRoe-ncv scheme (7-11) together with (12) and variable $Y^{t}=(U, P, g(\rho, s), C, \psi)$ perfectly preserves contact discontinuities, in the sense of definition 1 .

\section{ONE-DIMENSIONAL NUMERICAL RESULTS}

We focus here on the behaviour of the schemes when at least one shock wave occurs in the solution. A detailed investigation of the rates of convergence of the basic approximate Godunov scheme is available $\mathrm{in}^{12}$, when restricting to EOS in $T_{1}$ such as the perfect gas state law or the Tamman EOS. In addition the rate of convergence of variables which are affected by the contact discontinuity such as the mean concentration is clearly $\frac{1}{2}\left(\mathrm{see}^{12}\right)$. Numerical tests have been obtained using $Y^{t}=(U, P, \rho, C, \psi)$. For more details on the $1 \mathrm{D}$ procedure, we refer to ${ }^{13}$. Most of the test cases were inspired by numerical experiments arising in the literature $\left({ }^{26},{ }^{24},{ }^{1},{ }^{27}\right)$.

EOS in the second class We start with results obtained using the stiffened gas EOS, which has been widely investigated by Abgrall and co-workers ${ }^{1},{ }^{27}$. The most straightforward decomposition leads to: $\rho e=\phi_{2}(P, C, \psi)=f_{2}(C, \psi) P+g_{2}(C, \psi)$ with: $f_{2}(C, \psi)=\frac{1}{\gamma(\psi)-1}$ and: $g_{2}(C, \psi)=\frac{P_{\text {inf }}(\psi)}{\gamma(\psi)-1}$. Thus $\phi_{1}(P, \rho, C, \psi)=\phi_{3}(P, \rho, C, \psi)=0$. Figure 
1 provides the measure of the $L^{1}$ norm of the error when computing a pure right-going 3 -shock wave followed by a contact discontinuity, with help of the hybrid scheme (7-11), (12). The hybrid scheme induces a rate of convergence close to $\frac{1}{2}$ for the density $\rho$, and 1 for both variables $U$ and $P$. The rate of convergence for the conservative VFRoe-ncv scheme $((7)$ with $(5)$, and $\left.Y^{t}=(U, P, \rho, C, \psi)\right)$ is approximately $\frac{1}{2}$ for all variables. The coarser and finer meshes respectively contain 100 regular cells and 160000 cells.

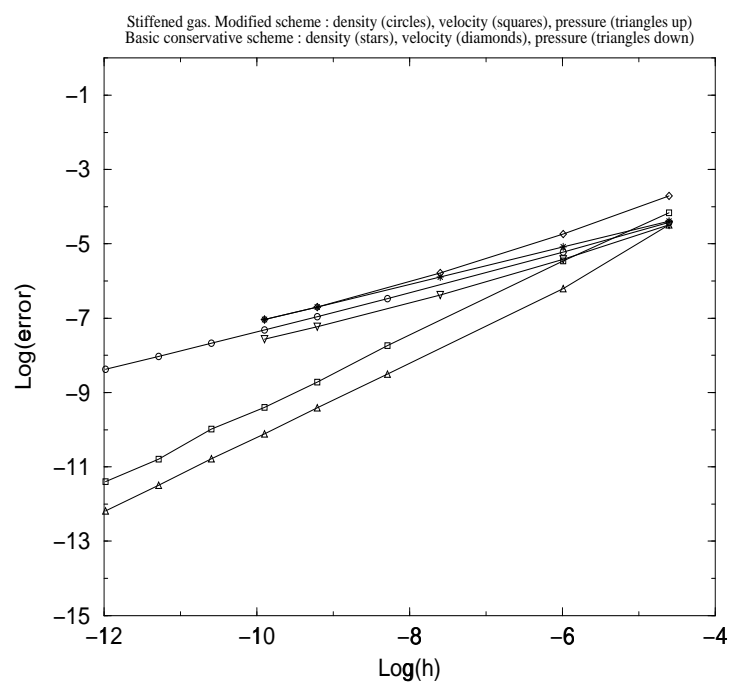

FIG. 1: Pure unsteady 3-shock wave - stiffened gas EOS - $L^{1}$ error norm

EOS in the third class The next example is devoted to Van der Waals EOS $\left(\mathrm{see}^{28}\right)$. In this case, the decomposition reads: $\rho e=\phi_{1}(P, \rho, C, \psi)+$ $\phi_{3}(P, \rho, C, \psi)$ with $\phi_{2}(P, C, \psi)=0$, where $b_{1}(P)=$ $c_{1}(P)=0, d_{1}(P)=\frac{P}{\gamma-1}, a_{1}(P)=\frac{-b P}{\gamma-1}$, and $\phi_{3}(P, \rho, C, \psi)=a \rho^{2}(-b \rho+2-\gamma)(\gamma-1)^{-1}$. The measure of the $L^{1}$ norm of the error, which is obtained by computing a 3 shock wave with the hybrid scheme and the basic conservative scheme (for which the rate of convergence is still $\frac{1}{2}$ for $\rho, U$, $P$ ), is provided in figure 2 . Regular meshes contain from 100 to 20000 cells. It thus clearly proved that approximate solutions including shocks such as those described in ${ }^{28}$ may not converge towards the right solution when applying for the hybrid scheme. Convergence to the right shock solution needs for instance some modification such as the one presented $\mathrm{in}^{13}$.

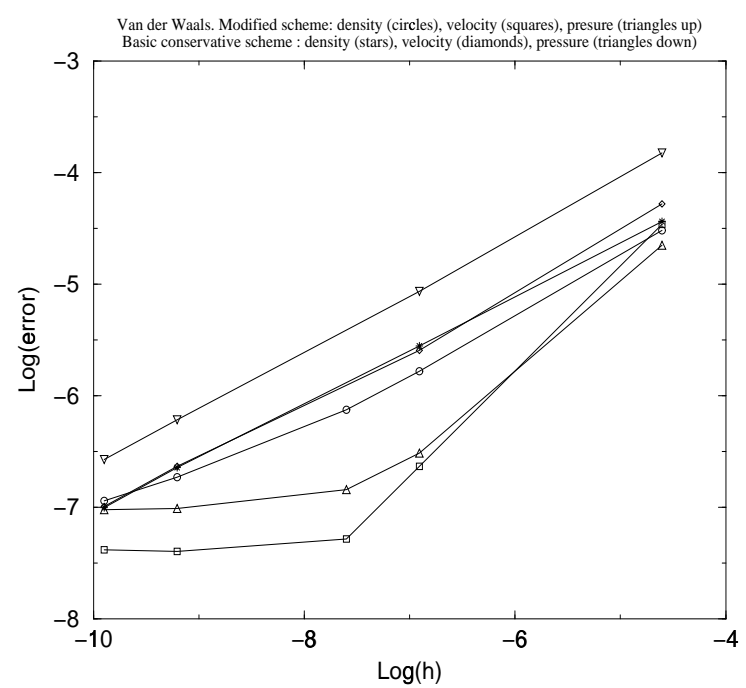

Fig. 2: Pure unsteady 3-shock wave - Van der Waals EOS - $L^{1}$ error norm

\section{Modified Godunov SCHEME IN A 2D FRAMEWORK}

\section{Introduction}

We may first introduce a preliminary step in which we examine the behaviour of the one dimensional evolution of a conservative $2 \mathrm{D}$ variable. This means that we focus on the conservative variable: $W=(\rho, \rho U, \rho V, E, \rho C, \rho \psi)$ and the governing set of equations is:

$$
\left\{\begin{array}{l}
\frac{\partial W}{\partial t}+\frac{\partial F(W)}{\partial x}=0 \\
W(x, 0)=W_{0}(x)
\end{array}\right.
$$

where $F(W)=\left(\rho U, \rho U^{2}+P, \rho U V, U(E+\right.$ $P), \rho U C, \rho U \psi$ ) denotes the physical flux. (U and $\mathrm{V}$ respectively stand for the velocity components in the $x$ and $y$ directions). This corresponds to the one dimensional Riemann problem which is solved at each interface of control volumes in a $2 \mathrm{D}$ framework. Thus, the total energy is now: $E=\rho U^{2} / 2+\rho V^{2} / 2+\rho e$.

We now assume that the initial condition of the Riemann problem is such that : $P_{L}=P_{R}=P_{0}$ and : $U_{L}=U_{R}=U_{0} ;$ moreover we take $\rho_{L}=\rho_{R}$ and $C_{L}=C_{R}, \psi_{L}=\psi_{R}$, but assume some strong shear: $V_{L}=-V_{R}=V_{0}$. This initial structure ensures that a pure moving contact discontinuity will travel to the right $\left(U_{0}>0\right)$, and hence : $W(x, t>0)=W_{0}\left(x-U_{0} t\right)$ for all $x$ in $\mathbb{R}$ and $t>0$. Unfortunately, even when restricting to perfect gas EOS (which lies in $T_{1}$ ), $\rho e=\frac{P}{\gamma-1}$, the conservative scheme will 
develop solutions which do not comply with this structure since the cell pressure predicted on the first cell on the right side of the initial discontinuity, will agree with $(\lambda=\delta t / h): U^{1}=U_{0}$ and $P^{1}=P_{0}+2(\gamma-1)\left(\rho_{0}\left(V_{0}\right)^{2}\left(1-\lambda U_{0}\right) \lambda U_{0}\right)$ ( $\delta t$ and $h$ still denote the time step and the size of a regular mesh respectively). Thus, though the pure conservative scheme still converges towards the right weak solution, the numerical approximations on coarse meshes will be polluted by strong shear flows. We will now show how to simply get rid of these numerical perturbations.

We still decompose the internal energy using (2$4)$. We then rewrite the total energy $E$ as:

$$
\begin{aligned}
E= & \rho U^{2} / 2+\phi_{1}(P, \rho, C, \psi)+\phi_{2}(P, C, \psi)(15) \\
& +\left(\phi_{3}(P, \rho, C, \psi)+\rho V^{2} / 2\right) .
\end{aligned}
$$

Thus, we may apply for the generic algorithm replacing $\phi_{3}$ by its counterpart $\varphi_{3}$ defined as:

$$
\varphi_{3}(P, \rho, C, \psi, V)=\phi_{3}(P, \rho, C, \psi)+\rho V^{2} / 2
$$

The governing equation of $\varphi_{3}$ is:

$$
\frac{\partial \varphi_{3}}{\partial t}+\frac{\partial U \varphi_{3}}{\partial x}+\left(\gamma P \frac{\partial \phi_{3}}{\partial P}+\rho \frac{\partial \phi_{3}}{\partial \rho}-\phi_{3}\right) \frac{\partial U}{\partial x}=0
$$

The moving contact discontinuity will be perfectly preserved in the following sense:

Property 2: For any EOS, if: $U_{k}^{n}=U_{0}$ and $p_{k}^{n}=P_{0}$ for $k=i-1, i, i+1$, the corresponding modified Godunov scheme or the modified approximate Godunov scheme VFRoe with variable $Y^{t}=(U, V, P, g(\rho, s), C, \psi)$ ensure that $p_{i}^{n+1}=P_{0}$, and $U_{i}^{n+1}=U_{0}$.

At this stage, we first note that even when focusing on the perfect gas EOS, the scheme will no longer converge towards the right weak solution, as soon as some shock is contained in the computational domain ( $\mathrm{see}^{17}$ for a similar result in the scalar case). We nonetheless recall that the amount of error on industrial meshes is so small when compared to the one occuring with the "good" conservative scheme, that the blended scheme described in $^{13}$ is obviously quite appealing. A second point is that the formulation (16) is not invariant under frame rotation. We thus now turn to the real 2D framework.

\section{The 2D framework}

Given some EOS, the standard decomposition is performed, using notations from (2-4).

$$
\rho e=\phi_{1}(P, \rho, C, \psi)+\phi_{2}(P, C, \psi)+\phi_{3}(P, \rho, C, \psi)
$$

The governing equations are now:

$$
\left\{\begin{array}{l}
\frac{\partial W}{\partial t}+\frac{\partial F(W)}{\partial x}+\frac{\partial G(W)}{\partial y}=0 \\
W(x, y, 0)=W_{0}(x, y)
\end{array}\right.
$$

The conservative variable is: $W=$ $(\rho, \rho U, \rho V, E, \rho C, \rho \psi)$. Convective fluxes are: $F(W)=\left(\rho U, \rho U^{2}+P, \rho U V, U(E+P), \rho U C, \rho U \psi\right)$, $G(W)=\left(\rho V, \rho U V, \rho V^{2}+P, V(E+P), \rho V C, \rho V \psi\right)$. We define $\mathbf{U}=(U, V)$ and introduce :

$\tilde{\phi}_{3}(P, \rho, U, V, C, \psi)=\phi_{3}(P, \rho, C, \psi)+\frac{\rho\left(U^{2}+V^{2}\right)}{2}$

Unlike in the previous section, $\tilde{\phi}_{3}$ is invariant under frame rotation. Actually, the total energy now reads:

$E=\phi_{1}(P, \rho, C, \psi)+\phi_{2}(P, C, \psi)+\tilde{\phi}_{3}(P, \rho, U, V, C, \psi)$

and the basic idea is to derive and compute in a suitable way the governing equation for $\tilde{\phi}_{3}$ instead of $\phi_{3}$. As a consequence, the appropriate definition of $p_{i}^{n}$ will issue from (23) instead of its counterpart (12) in a pure $1 D$ framework.

In the scheme below, $\mathcal{V}(i)$ refers to the indexes of the neighbouring cells of a cell $\Omega_{i}$, $\delta t$ still denotes the time step, $S_{i j}$ stands for the length of interface between cells $\Omega_{i}$ and $\Omega_{j}$, and $n_{i j}=\left(n_{x}, n_{y}\right)_{i j}$ is the unit outward normal vector pointing to cell $\Omega_{j}$. We also note $n^{t}=\left(n_{x}, n_{y}\right)$ with $\left(n_{x}\right)^{2}+\left(n_{y}\right)^{2}=1$. For any EOS which does not lie in $T_{1}$, we still set: $g_{0}(C, \psi)=f_{2}(C, \psi) h_{2}\left(P_{\text {ref }}\right)+g_{2}(C, \psi)$.

We also need to define 'perfect local preservation of contact discontinuities' when computing Euler equations on triangular meshes:

Definition 2: We consider a triangular mesh, and a contact discontinuity travelling in the $n$ direction. We suppose that some cell $\Omega_{i}$ has an interface $i j_{0}$ (triangle $\Omega_{j_{0}}$ is a neighbour of $\left.\Omega_{i}\right)$ which is aligned with $n: n_{i j_{0}} . n=1$. We assume that: $\left(\mathbf{U}_{k}^{n}\right) \cdot n=U_{0}, p_{k}^{n}=P_{0}$, for $k$ in $i \cup \mathcal{V}(i)$, but also that: $\left(\mathbf{U}_{k}^{n}\right) \cdot \tau=V_{l},(\rho)_{k}^{n}=\rho_{l}$, $C_{k}^{n}=C_{l},(\psi)_{k}^{n}=\psi_{l}$ if the interface $i k$ is on the left side of the interface $i j_{0}$, and $\left(\mathbf{U}_{k}^{n}\right) \cdot \tau=V_{r}$, 
$(\rho)_{k}^{n}=\rho_{r}, C_{k}^{n}=C_{r},(\psi)_{k}^{n}=\psi_{r}$ if interface $i k$ is on the right side of the interface $i j_{0}$. Then, a first-order four-point scheme will perfectly preserve local contact discontinuities travelling in the $n$ direction, if it computes $p_{i}^{n+1}=P_{0}$, and $\left(\mathbf{U}_{i}^{n+1}\right) \cdot n=U_{0}$ when using the previous data.

We now introduce the 2D first-order scheme:

$$
\begin{aligned}
& \operatorname{mes}\left(\Omega_{i}\right)\left(W_{i}^{n+1}-W_{i}^{n}\right) \\
& +\delta t \sum_{j \in \mathcal{V}(i)}\left(\left(n_{x} F+n_{y} G\right)\left(W\left(Y_{i j}^{*}\right), n_{i j}\right) S_{i j}\right)=0 \\
& \operatorname{mes}\left(\Omega_{i}\right)\left(\left(g_{0}\right)_{i}^{n+1}-\left(g_{0}\right)_{i}^{n}\right) \\
& +\delta t\left(\sum_{j \in \mathcal{V}(i)}\left(g_{0}\right)_{i j}^{*}(\mathbf{U} \cdot n)_{i j}^{*} S_{i j}\right) \\
& -\delta t \hat{g_{0}}\left(\sum_{j \in \mathcal{V}(i)}(\mathbf{U} \cdot n)_{i j}^{*} S_{i j}\right)=0 \\
& \operatorname{mes}\left(\Omega_{i}\right)\left(\left(\tilde{\phi}_{3}\right)_{i}^{n+1}-\left(\tilde{\phi}_{3}\right)_{i}^{n}\right) \\
& \quad+\delta t\left(\sum_{j \in \mathcal{V}(i)}\left(\left(P_{i j}^{*}+\left(\tilde{\phi}_{3}\right)_{i j}^{*}\right)(\mathbf{U} . n)_{i j}^{*} S_{i j}\right)\right. \\
& +\delta t \hat{H}_{i}\left(\sum_{j \in \mathcal{V}(i)}(\mathbf{U} . n)_{i j}^{*} S_{i j}\right)=0 \\
& \operatorname{card}(\mathcal{V}(i)) \hat{H}_{i}= \\
& \sum_{j \in \mathcal{V}(i)}\left(\gamma P \frac{\partial \phi_{3}}{\partial P}+\rho \frac{\partial \phi_{3}}{\partial \rho}-\phi_{3}-P\right)_{i j}^{*} \\
& \operatorname{card}(\mathcal{V}(i)){\hat{g_{0}}}_{i}=\sum_{j \in \mathcal{V}(i)}\left(g_{0}\right)_{i j}^{*}
\end{aligned}
$$

The cell pressure $p_{i}^{n+1}=\tilde{P}_{i}^{n+1}$ is computed solving :

$$
\begin{aligned}
E_{i}^{n+1}= & \phi_{1}\left(\tilde{P}_{i}^{n+1}, \rho_{i}^{n+1}, C_{i}^{n+1}, \psi_{i}^{n+1}\right) \\
& +\left(f_{2}\right)_{i}^{n+1} h_{2}\left(\tilde{P}_{i}^{n+1}\right)+\left(g_{2}\right)_{i}^{n+1} \\
& +\left(\tilde{\phi}_{3}\right)_{i}^{n+1}
\end{aligned}
$$

Obviously, the following one-dimensional Riemann problem needs to be solved at each interface between cells $\Omega_{i}$ and $\Omega_{j}$, while noting $J\left(W_{n}\right)=$ $\left(\rho U_{n}, \rho\left(U_{n}\right)^{2}+P, \rho U_{n} U_{\tau}, U_{n}(E+P), \rho U_{n} C, \rho U_{n} \psi\right)$, $W_{n}=\left(\rho, \rho U_{n}, \rho U_{\tau}, E, \rho C, \rho \psi\right), U_{n}=n_{x} U+n_{y} V$, $U_{\tau}=-n_{y} U+n_{x} V$, and $n_{i j}$ the unit normal vector pointing from cell $\Omega_{i}$ to cell $\Omega_{j}$ :

$$
\left\{\begin{array}{l}
\frac{\partial W_{n}}{\partial t}+\frac{\partial J\left(W_{n}\right)}{\partial n}=0 \\
W_{n}\left(x . n_{i j}<0, t=0\right)=\left(W_{n}\right)_{i}^{n} \\
W_{n}\left(x . n_{i j}>0, t=0\right)=\left(W_{n}\right)_{j}^{n}
\end{array}\right.
$$

When restricting to perfect gas EOS or stiffened gas EOS, note that $H=-P$ since (20) corresponds to the governing equation of kinetic energy.

Property 3: For any EOS, the modified Godunov scheme and the modified Godunov scheme VFRoe based on (18)-(22) with variable $Y^{t}=$ $(U, V, P, g(\rho, s), C, \psi)$ perfectly preserve local contact discontinuities in the sense of definition 2 .

In other words, when the mesh interfaces are aligned with the local slip line, the profiles are "perfectly" accounted for by the scheme. This seems at first glance to be in favour of meshes which have a great number of faces, rather than triangles. These initial conditions may thus contain some rather strong shear. The property is no longer true when none among the interfaces of cell $\Omega_{i}$ is aligned with $n$. We may also wonder what happens when using more uniform initial condition on the velocity field. Actually, the above scheme obviously also enjoys the following property :

Property 4: For any EOS, and for given initial data in agreement with: $U_{k}^{n}=U_{0}, V_{k}^{n}=V_{0}$ and $p_{k}^{n}=P_{0}$ with $k$ in $i \cup \mathcal{V}(i)$, the modified Godunov scheme or the modified approximate Godunov scheme VFRoe based on (18)-(22) with variable $Y^{t}=(U, V, P, g(\rho, s), C, \psi)$ ensure that $p_{i}^{n+1}=P_{0}, U_{i}^{n+1}=U_{0}$, and $V_{i}^{n+1}=V_{0}$.

Another way to handle the $2 \mathrm{D}$ computation consists in using a straightforward extension of the original scheme (7)-(11) (this may be easily checked using the rule $\Delta(a b)=\bar{a} \Delta(b)+\bar{b} \Delta(a)$, setting $\Delta \alpha=\alpha_{r}-\alpha_{l}$ and $\left.\bar{\alpha}=\left(\alpha_{r}+\alpha_{l}\right) / 2\right)$ :

$$
\begin{aligned}
& \operatorname{mes}\left(\Omega_{i}\right)\left(W_{i}^{n+1}-W_{i}^{n}\right) \\
& +\delta t \sum_{j \in \mathcal{V}(i)}\left(\left(n_{x} F+n_{y} G\right)\left(W\left(Y_{i j}^{*}\right), n_{i j}\right) S_{i j}\right)=0 \\
& \operatorname{mes}\left(\Omega_{i}\right)\left(\left(g_{0}\right)_{i}^{n+1}-\left(g_{0}\right)_{i}^{n}\right) \\
& +\delta t\left(\sum_{j \in \mathcal{V}(i)}\left(g_{0}\right)_{i j}^{*}(\mathbf{U} . n)_{i j}^{*} S_{i j}\right) \\
& -\delta t \hat{g}_{0}\left(\sum_{j \in \mathcal{V}(i)}(\mathbf{U} . n)_{i j}^{*} S_{i j}\right)=0 \\
& \operatorname{mes}\left(\Omega_{i}\right)\left(\left(\phi_{3}\right)_{i}^{n+1}-\left(\phi_{3}\right)_{i}^{n}\right) \\
& +\delta t\left(\sum_{j \in \mathcal{V}(i)}\left(\left(\phi_{3}\right)_{i j}^{*}(\mathbf{U} . n)_{i j}^{*} S_{i j}\right)\right. \\
& +\delta t \hat{H}_{i}\left(\sum_{j \in \mathcal{V}(i)}(\mathbf{U} . n)_{i j}^{*} S_{i j}\right)=0
\end{aligned}
$$

American Institute of Aeronautics and Astronautics 
using the notations:

$$
\begin{aligned}
& \operatorname{card}(\mathcal{V}(i)) \hat{H}_{i}= \\
& \sum_{j \in \mathcal{V}(i)}\left(\gamma P \frac{\partial \phi_{3}}{\partial P}+\rho \frac{\partial \phi_{3}}{\partial \rho}-\phi_{3}\right)_{i j}^{*} \\
& \operatorname{card}(\mathcal{V}(i)) \hat{g}_{0}=\sum_{j \in \mathcal{V}(i)}\left(g_{0}\right)_{i j}^{*}
\end{aligned}
$$

The cell pressure $p_{i}^{n+1}=\tilde{P}_{i}^{n+1}$ is now computed solving :

$$
\begin{aligned}
E_{i}^{n+1}= & \frac{\rho_{i}^{n+1}}{2}\left(\left(U_{i}^{n+1}\right)^{2}+\left(V_{i}^{n+1}\right)^{2}\right) \\
& \phi_{1}\left(\tilde{P}_{i}^{n+1}, \rho_{i}^{n+1}, C_{i}^{n+1}, \psi_{i}^{n+1}\right) \\
& +\left(f_{2}\right)_{i}^{n+1} h_{2}\left(\tilde{P}_{i}^{n+1}\right)+\left(g_{2}\right)_{i}^{n+1} \\
& +\left(\phi_{3}\right)_{i}^{n+1}
\end{aligned}
$$

Property 5: For any EOS, and for given initial data in agreement with: $U_{k}^{n}=U_{0}, V_{k}^{n}=V_{0}$ and $p_{k}^{n}=P_{0}$ with $k$ in $i \cup \mathcal{V}(i)$, the modified Godunov scheme or the modified approximate Godunov scheme VFRoe based on (25)-(29) with variable $Y^{t}=(U, V, P, g(\rho, s), C, \psi)$ ensure that $p_{i}^{n+1}=P_{0}, U_{i}^{n+1}=U_{0}$, and $V_{i}^{n+1}=V_{0}$.

The scheme ((25)-(29)) clearly does not preserve local contact discontinuities (as defined in definition 2) as the former ((18)-(22)) does.

\section{D NUMERICAL RESULTS}

Computational results given below for the first three test cases have been obtained using the hybrid scheme (18-22) and the fully conservative scheme. The computational domain is $[0,1] \times[0,1]$. It contains 3 nodes in the $y$-direction when focusing on the first three test cases since the mesh contains rectangular cells. The number of mesh points refers to the number of cells in the $x$ direction. The $C F L$ number is always set to 0.5 , unless otherwise specified. Results are plotted along the straight line $y=0.5$. The fourth test case involves triangular meshes.

\section{Plane travelling contact discontinuity with strong shear}

We show below results obtained using the perfect gas EOS in a 2D framework. Initial conditions on each side of the line $x=0.5$ are: $U_{L}=100, V_{L}=-100, P_{L}=10^{5}, \rho_{L}=1$, and : $U_{R}=100, V_{R}=50, P_{R}=10^{5}, \rho_{R}=2$. As expected, the axial velocity and the pressure remain uniform in the whole computational domain when applying for the hybrid scheme.

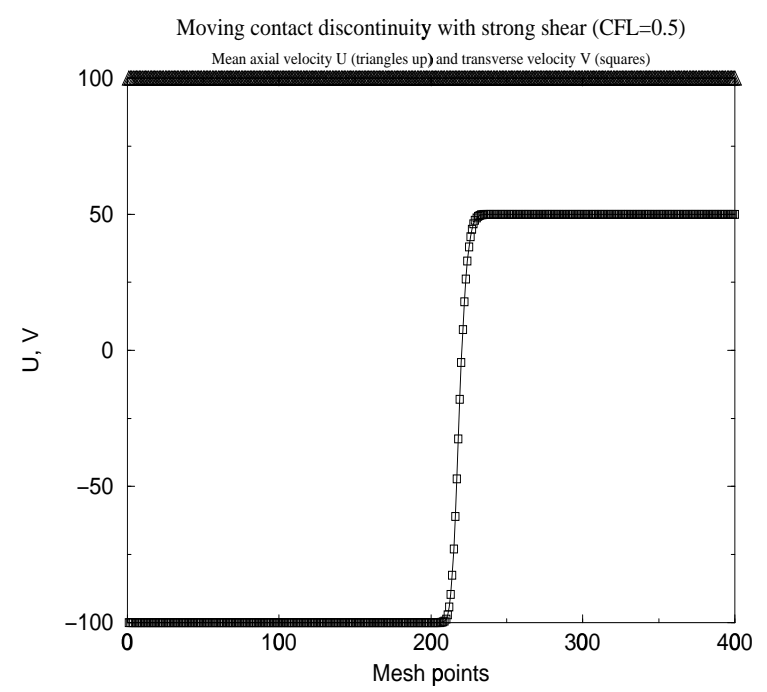

FIG. 3: Hybrid scheme with strong shear -coarse mesh-

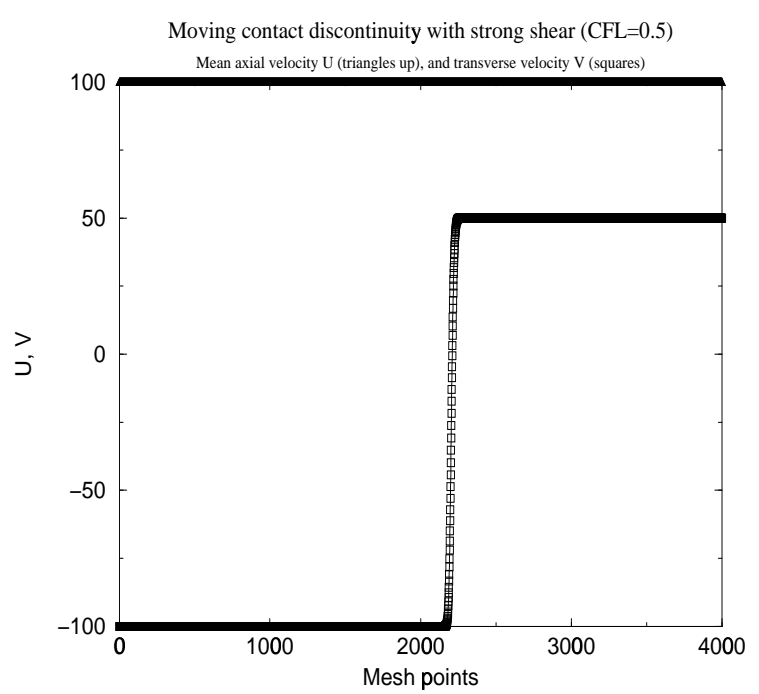

FIG. 4: Hybrid scheme with strong shear -fine mesh- 


\section{The "odd-even" test case}

We now assume some travelling contact discontinuity, that is: $U_{L}=100, P_{L}=10^{5}, \rho_{L}=1$, and : $U_{R}=100, P_{R}=10^{5}, \rho_{R}=2$ on the left and right side of the initial membrane located at $x=0.5$. Besides, the transverse velocity obeys: $V_{i, j}+V_{i+1, j}=0$, with $V_{1, j}=100$. The coarse mesh and the fine mesh contain 400 and 4000 uniform cells respectively in the $x$ direction. Figures 5 and 6 show the computed solution at $t=T$ and $t=0+=\delta t$. It clearly appears that the initial odd-even decoupling is damped w.r.t. time. Once again, the axial velocity $U$ and the pressure $P$ remain uniform over the whole computational domain when using the hybrid scheme.

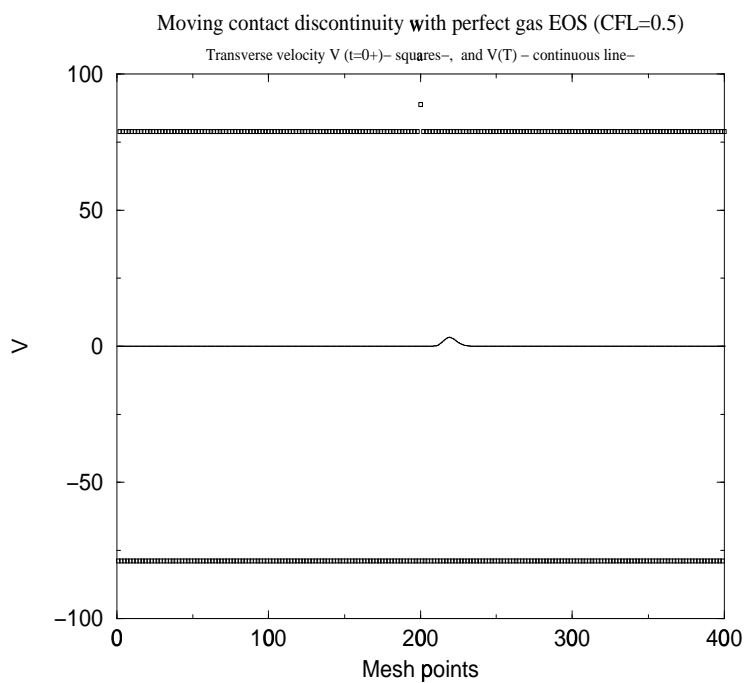

FIG. 5: Hybrid scheme with even-odd initial condition on $\mathrm{V}$ on coarse mesh

\section{Plane shock tube on a rectangular mesh}

Results on figures 7, 8, 9 correspond to a shock tube aparatus with initial data generating a strong supersonic shock tube with strong initial shear on $\mathrm{V}$. These are : $U_{L}=0, V_{L}=-100, P_{L}=$ $10^{5}, \rho_{L}=1$ and $U_{R}=0, V_{R}=50, P_{R}=10^{3}, \rho_{R}=$ 0.0125 apart from $x=0.5$. The time step is still in agreement with $C F L=0.5$. Computational results on a fine mesh with 8000 cells in the $x$ direction enable to check that the behaviour of the sonic point is not altered, and that both the velocity and the pressure remain almost uniform around the moving contact discontinuity, which is easly located by variations of transverse velocity. Results below show both approximations obtained using the hybrid scheme or the conservative either. Obviously, the conservative scheme provides

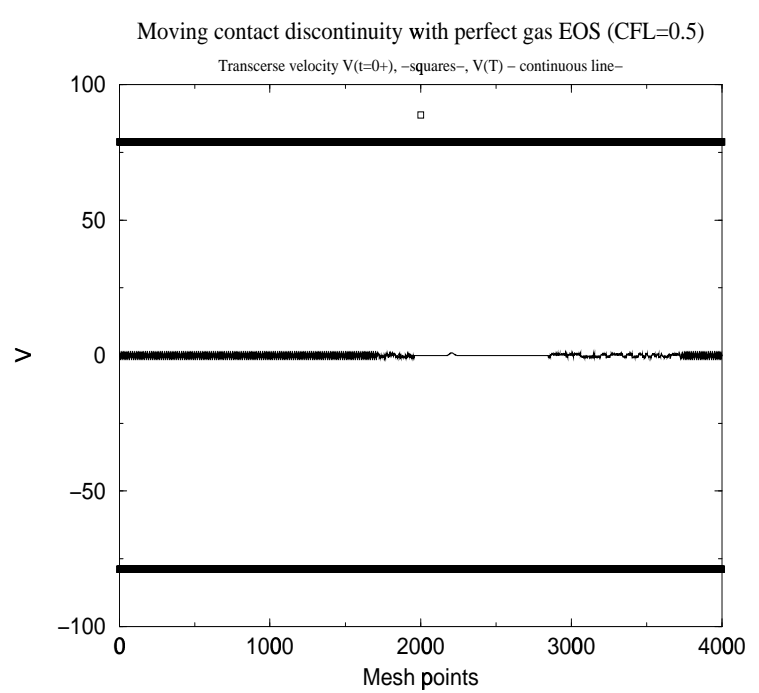

FIG. 6: Hybrid scheme with even-odd initial condition on $\mathrm{V}$ on fine mesh

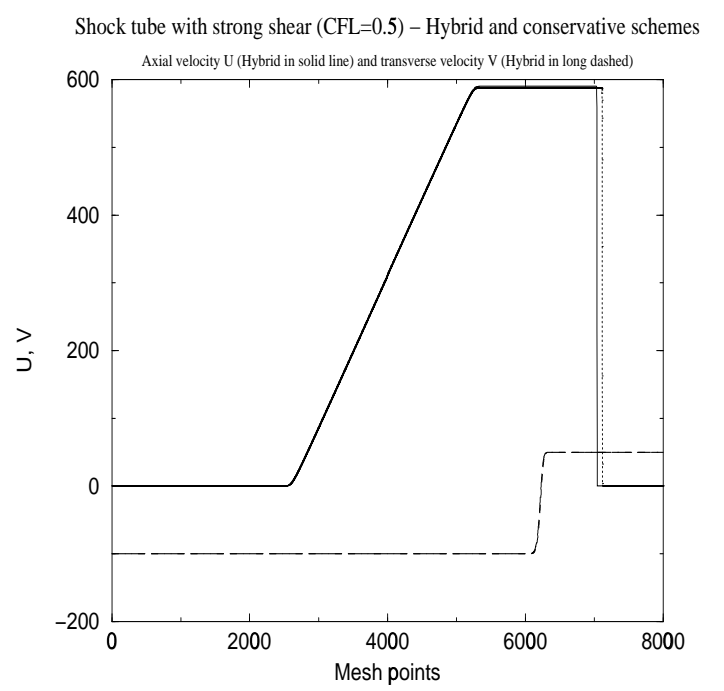

FIG. 7: Hybrid scheme and conservative scheme velocity in shock tube with strong shear on fine mesh 


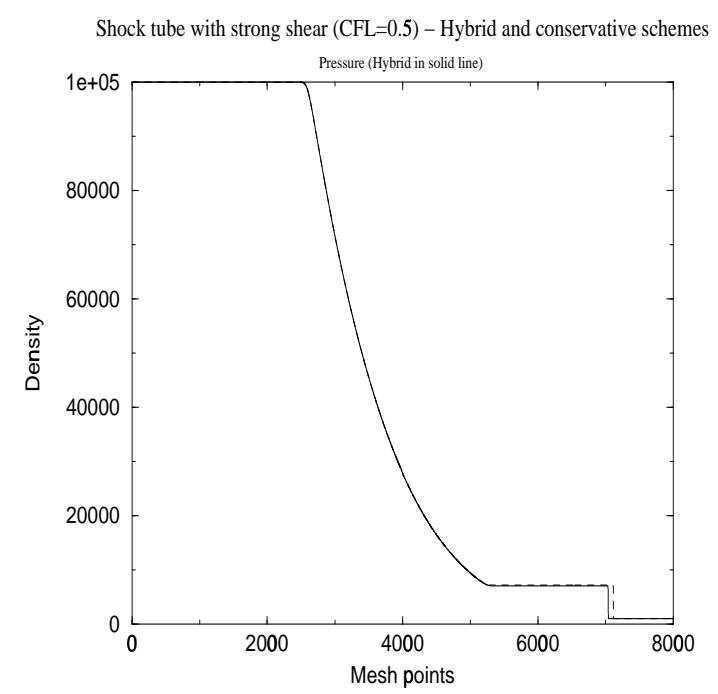

FIG. 8: Hybrid scheme and conservative scheme pressure in shock tube with strong shear on fine mesh

approximations of both $U, P$ which only slightly vary through the contact discontinuity. On the other side, one may notice that the occurence of the shock wave inhibits the convergence of the hybrid scheme towards the right solution.

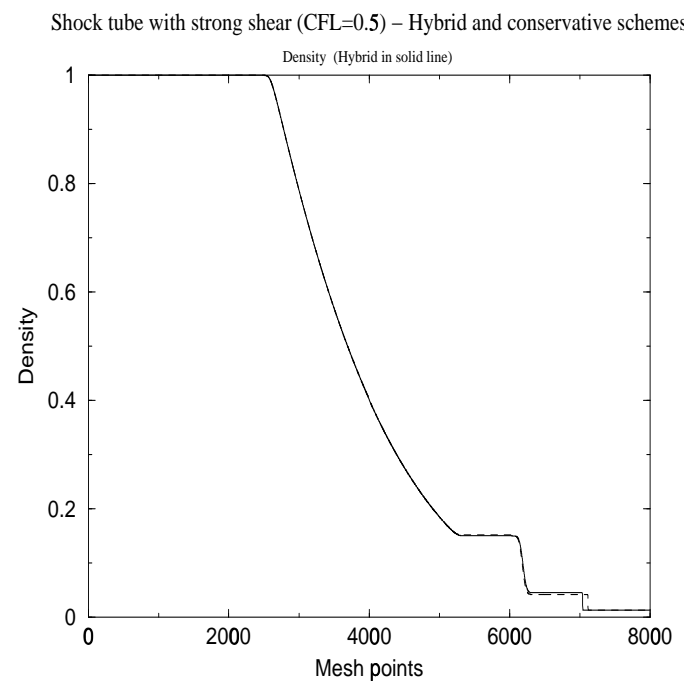

FIG. 9: Hybrid scheme and conservative scheme density in shock tube with strong shear on fine mesh

Plane travelling contact discontinuity on a triangular mesh

Results presented in figure 10, correspond to the measure of the $L^{1}$ error norm when computing a plane contact discontinuity with strong shear ef- fects on a triangular mesh with the conservative scheme. On the basis of previous remarks, we expect to find a convergence rate $h^{0.5}$ for both velocity and pressure variables. The domain is now : $\Omega=[0,2] \times[0,2]$. The initial conditions apart from the axis $x=0.5$ are: $U_{L}=100, V_{L}=-100, P_{L}=$ $10^{5}, \rho_{L}=1, U_{R}=100, V_{R}=50, P_{R}=10^{5}, \rho_{R}=$ 2. The CFL number is 0.5 . Triangular meshes include from 1300 up to 1331200 cells.

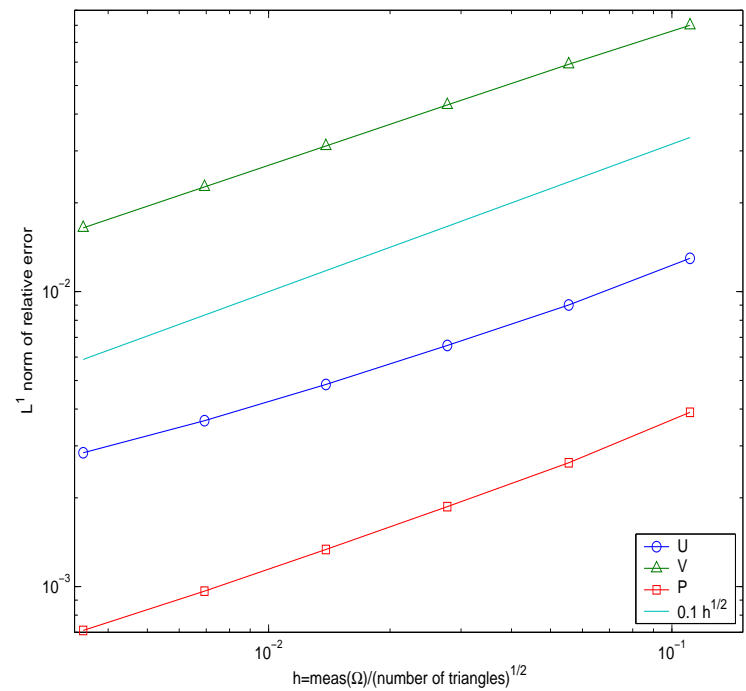

FIG. 10: Conservative scheme - Error norm for pressure and for axial velocity

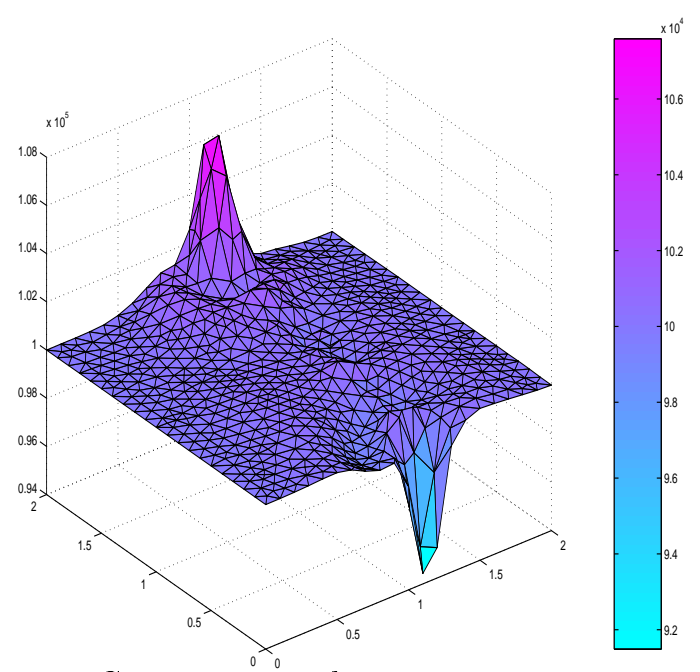

FIG. 11: Conservative scheme - Moving contact discontinuity- Cell values of pressure

\section{Conclusion}

The hybrid scheme is thus useful both in the 1D and 2D framework. Furthermore, the blended 


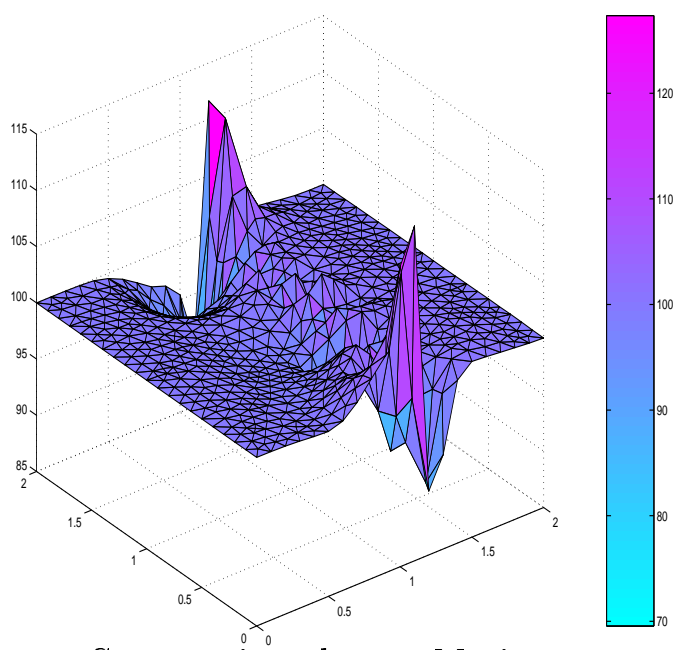

FIG. 12: Conservative scheme - Moving contact discontinuity- Cell values of axial velocity

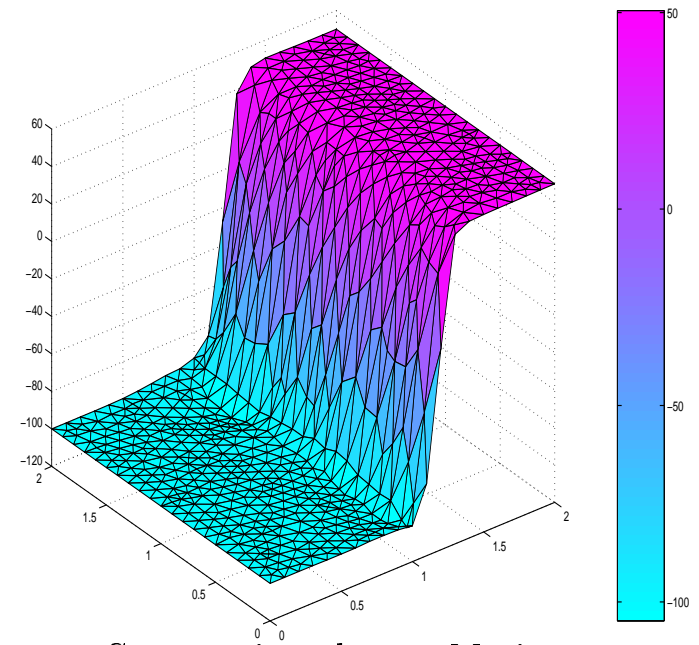

FIG. 13: Conservative scheme - Moving contact discontinuity- Cell values of transverse vellocity scheme which turns off the hybrid scheme when the mesh size tends to 0 :

$$
p_{i}^{n}=\alpha(h) \tilde{P}_{i}^{n}+(1-\alpha(h)) P_{i}^{n}
$$

-where $\tilde{P}_{i}^{n}$ issues from (23)- enables to get rid of small deficiencies of the hybrid scheme on very fine meshes (that is non convergence towards the right shock solution). A point that is not obvious from a theoretical point of view is how to define an optimal smoothing function $\alpha(h)$ which agrees with $\alpha(h=+\infty)=1$ and $\alpha(h=0)=0$, which would minimize the error on any mesh. This may be achieved in academic conditions, but this is more tough in a practical computation. Nonetheless, we emphasize that this is not a real problem nowadays, since the error of the hybrid scheme on industrial meshes (thus including at most a few hundred points in each direction) is indeed much lower than the error associated with the conservative scheme. This however should probably be reconsidered in a few years, owing to the increasing capacity of computers.

Acknowledgments: the second author has benefited from financial support through the CEA-EDF project NEPTUNE.

\section{REFERENCES}

[1] R. Abgrall, How to prevent pressure oscillations in multicomponent flow calculations: a quasi conservative approach, J. Comp. Phys., vol. 125, pp. 150-160, 1995.

[2] R. Abgrall and S. Karni, Computations of compressible multifluids, J. Comp. Phys., vol. 169 , pp. 594-623, 2001.

[3] R. Abgrall, B. Nkonga and R. Saurel, Efficient numerical approximation of compressible multi-material flow for unstructured meshes, Computers and Fluids, vol. 32-4, pp. 571-606, 2002.

[4] G. Allaire, S. Clerc and S. Kokh, A five equation model for the simulation of interfaces between compressible fluids, J. Comp. Phys., vol. 181, pp. 577-616, 2002.

[5] M.R. Baer and J.WS. Nunziato, A two phase mixture theory for the deflagration to detonation (DDT) transition in reactive flows, Int. J. of Multiphase Flow, vol. 12-6, pp. 861-889, 1986.

10

American Institute of Aeronautics and Astronautics 
[6] T. Barberon, P. Helluy, Finite Volume simulations of cavitating flows, Proceedings of $\mathrm{Fi}$ nite Volumes for Complex Applications III, $R$. Herbin and D. Kroner editors, Hermes Penton Science, pp. 455-464, 2002.

[7] S. Bilicki and J. Kestin, Physical aspects of the relaxation model in two phase flows, Proc. of the Royal Soc. of London, vol. A428, pp. 379-397, 1990.

[8] T. Buffard, T. Gallouët and J.M. Hérard, A sequel to a rough Godunov scheme. Application to real gas flows, Computers and Fluids, vol. 29-7, pp. 813-847, 2000.

[9] S. Clerc, Numerical simulation of the homogeneous equilibrium model for two phase flows, J. Comp. Phys., vol. 161-1, pp. 354375,2000 .

[10] F. Coquel, T. Gallouët, J.M. Hérard and N. Seguin, Closure laws for a two-fluid two pressure model, C. R. Acad. Sci. Paris, vol. I334, pp. 927-932, 2002.

[11] R.P. Fedkiw, T. Aslam, B. Merriman and S. Osher, A non oscillatory eulerian approach to interfaces in multimaterial flows (the ghost fluid approach), J. Comp. Phys., vol. 152, p. $457,1999$.

[12] T. Gallouët, J.M. Hérard and N. Seguin, Some recent Finite Volume schemes to compute Euler equations using real gas EOS, Int. J. for Num. Meth. in Fluids, vol. 39, pp. 1073$1138,2002$.

[13] — A hybrid scheme to compute contact discontinuities in one dimensional Euler systems, Math. Modelling and Numerical Analysis, vol. 36, pp.1133-1159, 2002 .

[14] - On the use of some symmetrizing variables to deal with vacuum, submitted for publication, 2002.

[15] E. Godlewski and P.A. Raviart, Numerical approximation for hyperbolic systems of conservation laws, Springer Verlag, 1996.

[16] S.K. Godunov, A difference method for numerical calculation of discontinous equations of hydrodynamics, Sbornik, pp. 271-300, 1959. In Russian.
[17] X. Hou and P. G. Le FLoch, Why non conservative schemes converge to wrong solutions, Mathematics of computation, vol. 62206, pp. 497-530, 1994.

[18] A.K. Kapila, S.F. Son, J.B. Bdzil, R. Menikoff and D.S. Stewart, Two-phase modelling of DDT : structure of the velocity relaxation zone, Physics of Fluids, vol. 9(12), pp. 3885-3897, 1997.

[19] S. Karni, Multicomponent flow calculations by a consistent primitive algorithm, J. Comp. Phys., vol. 112, pp. 31-43, 1994.

[20] - Hybrid multifluid algorithms, SIAM J. Sci. Comp., vol. 17, pp. 1019-1039, 1996.

[21] S. Karni and R. Abgrall, Ghost fluid for the poor: a single fluid algorithm for multifluid, Oberwolfach, 2001.

[22] R. Kee, J. Miller and T. Jefferson, Chemkin: a general purpose, problem independant transportable fortran chemical kinetics code package, SAND Report 80-8003, Sandia National Laboratories.

[23] N. Jullian, PhD thesis, Université de Provence, Marseille, France, in preparation.

[24] A. Letellier and A. Forestier, Le problème de Riemann en fluide quelconque, CEA-DMT Report 93/451, 1993. In French.

[25] R. LeVeque, Numerical methods for conservation laws, Birkhauser, 1992.

[26] S. Rouy, Modélisation mathématique et numérique d'écoulements diphasiques compressibles, $\mathrm{PhD}$ thesis, Université de Toulon et du Var, France, December 2000.

[27] R. Saurel and R. Abgrall, A simple method for compressible multifluid flows, SIAM $J$. Sci. Comp., vol. 21-3, pp. 1115-1145, 1999.

[28] K.M. Shyue, A fluid mixture type algorithm for compressible multicomponent flow with Van der Waals equation of state, J. Comp. Phys., vol. 156, pp. 43-88, 1999.

[29] J. Smoller, Shock waves and reaction diffusion equations, Springer Verlag, 1983.

[30] E.F. Toro, Riemann solvers and numerical methods for fluid dynamics, Springer Verlag, 1997.

\section{1}

American Institute of Aeronautics and Astronautics 